DOI 10.4171/JEMS/404

Michael Struwe

\title{
The critical nonlinear wave equation in two space dimensions
}

Received November 23, 2010

Abstract. Extending our previous work [9], we show that the Cauchy problem for wave equations with critical exponential nonlinearities in two space dimensions is globally well-posed for arbitrary smooth initial data.

\section{Introduction}

Consider the equation

$$
u_{t t}-\Delta u+u e^{u^{2}}=0 \quad \text { on } \mathbb{R} \times \mathbb{R}^{2} .
$$

In [2] Ibrahim, Majdoub, and Masmoudi demonstrated that the initial value problem for equation (1) is well-posed for smooth Cauchy data

$$
\left(u, u_{t}\right)_{t=0}=\left(u_{0}, u_{1}\right)
$$

with initial energy

$$
E(u(0))=\int_{\mathbb{R}^{2}} e(u(0)) d x \leq 2 \pi,
$$

where

$$
e(u)=\frac{1}{2}\left(\left|u_{t}\right|^{2}+|\nabla u|^{2}+e^{u^{2}}-1\right) .
$$

Equation (1) is closely related to the critical Sobolev embedding in two space dimensions defined by the Moser-Trudinger inequality

$$
\sup _{u \in H_{0}^{1}(\Omega),\|\nabla u\|_{L^{2}(\Omega)}^{2} \leq 1} \int_{\Omega} e^{\alpha u^{2}} d x \leq C(\alpha)|\Omega|
$$

for any bounded domain $\Omega \subset \mathbb{R}^{2}$ having 2-dimensional Lebesgue measure $|\Omega|$ and any $\alpha \leq 4 \pi$, with a constant $C(\alpha)<\infty$ independent of $\Omega$; see [6], [11]. For $\alpha>4 \pi$ the above supremum is infinite. In particular, when $E(u(0))>2 \pi$, not even a locally uniform spatial $L^{1}$-bound is available for the term $u e^{u^{2}}$. In analogy with nonlinear wave equations

$$
u_{t t}-\Delta u+u|u|^{p-2}=0 \quad \text { on } \mathbb{R} \times \mathbb{R}^{n}
$$

M. Struwe: Mathematik, ETH-Zürich, CH-8092 Zürich, Switzerland; e-mail: struwe@ math.ethz.ch 
with $p>2 n /(n-2)$ in $n \geq 3$ space dimensions, where the nonlinear term cannot be bounded in the dual space of $H^{1}$ in terms of the Dirichlet energy, the Cauchy problem for equation (1) was therefore termed "supercritical" for initial data with energy $E(u(0))>2 \pi$. The recent results [1], [3] of Ibrahim, Jrad, Majdoub, and Masmoudi, showing that the local solution of the Cauchy problem (1), (2) does not depend on the initial data in a locally uniformly continuous fashion when $E(u(0))>2 \pi$, seemed to further justify this classification.

However, in contrast with these results, in [9] we were able to show that the Cauchy problem (1), (2) is well-posed in the radially symmetric case, regardless of the size of the data. Here we show that the restriction (3) is not needed in the general case either.

Theorem 1.1. For any $u_{0}, u_{1} \in C^{\infty}\left(\mathbb{R}^{2}\right)$ there exists a unique, smooth solution $u$ to the Cauchy problem (1), (2), defined for all time.

The proof of Theorem 1.1 is strikingly different from the proof of the companion result in the spherically symmetric setting. In the latter case, locally uniform pointwise bounds for the solution away from $x=0$ permit one to rule out blow-up by means of standard multipliers. In contrast, in the present setting the usual multiplier technique only seems to give decay of the energy in the interior of any light cone, and full control only of certain components. In particular, we cannot rule out outgoing waves concentrating energy near the lateral boundary of the light cone. However, in combination with a subtle improvement of the Moser-Trudinger inequality (5), stated as Lemma 4.3 below, the partial control of the energy that we achieve allows us to improve the bounds for the nonlinear term in equation (1) sufficiently for ruling out blow-up. Lemma 4.3 may also be of interest in itself.

Note that no weighted energy estimates are required in the proof, as would be expected in a truly "supercritical" context. It thus appears that problem (1), (2) still belongs to the realm of "critical" equations. More generally, it seems that this may be true for all problems where smallness of the energy implies regularity, as in the present case. See [3], [5], [8], [10] for recent results on supercritical wave equations, and [7] for background material on nonlinear wave equations in general.

\section{Basic estimates}

For the proof of Theorem 1.1 we argue indirectly, as in [9]; that is, we suppose that the local solution $u$ to (1), (2) for certain Cauchy data $u_{0}, u_{1} \in C^{\infty}\left(\mathbb{R}^{2}\right)$ cannot be smoothly extended to a neighborhood of some point $\left(T_{0}, x_{0}\right)$ where $T_{0} \geq 0$. As shown in [9], we may assume that $u_{0}, u_{1}$ are compactly supported, $T_{0}>0$, and that $u \in C^{\infty}\left(\left[0, T_{0}\left[\times \mathbb{R}^{2}\right)\right.\right.$.

After translating the origin of our coordinate system to the point $x_{0}$, if necessary, we may assume that $x_{0}=0$. Also shifting time by $T_{0}$ and then reversing the arrow of time, in the following we may assume that we have a compactly supported solution $\left.\left.u \in C^{\infty}(] 0, T_{0}\right] \times \mathbb{R}^{2}\right)$ of (1) blowing up at $(0,0)$.

We now briefly recall some standard estimates from [9] that will also be needed for the present approach. 


\subsection{Energy inequality and flux decay}

Upon multiplying (1) by $u_{t}$ we obtain the conservation law

$$
0=\frac{d}{d t} e(u)-\operatorname{div}\left(\nabla u \cdot u_{t}\right)
$$

for the energy density $e(u)$ and the density of momentum

$$
m(u)=\nabla u \cdot u_{t} .
$$

In the following, we only will make use of equation (7) on compact regions. In order to simplify later computations, we therefore now drop the term -1 in the definition of $e(u)$ above and henceforth let

$$
e(u)=\frac{1}{2}\left(\left|u_{t}\right|^{2}+|\nabla u|^{2}+e^{u^{2}}\right) .
$$

The original definition (4) was made to ensure that compactly supported functions $u$ have finite total energy.

Since clearly $|m(u)| \leq e(u)$, integration of (7) over a truncated light cone yields

$$
E\left(u(t), B_{R}\left(x_{0}\right)\right):=\int_{B_{R}\left(x_{0}\right)} e(u(t)) d x \leq E\left(u(s+t), B_{R+|s|}\left(x_{0}\right)\right)
$$

for any $x_{0} \in \mathbb{R}^{2}, R>0$, and $0<s+t, t \leq T_{0}$. In particular, energy will spread with speed at most 1 .

Estimate (8) neglects the flux terms, which will be important later. Of particular interest will be the case when $x_{0}=0$. For $0<S \leq T \leq T_{0}$ denote by $v(y)=u(|y|, y)$ the restriction of $u$ to the lateral boundary

$$
M_{S}^{T}=\{z=(t, x) ; S \leq t \leq T,|x|=t\}
$$

of the truncated forward light cone

$$
K_{S}^{T}=\{z=(t, x) ; S \leq t \leq T,|x| \leq t\}
$$

with vertex at $z=(0,0)$. Upon integrating (7) over $K_{S}^{T}$ we then find the identity

$$
E\left(u(S), B_{S}(0)\right)+\operatorname{Flux}\left(u, M_{S}^{T}\right)=E\left(u(T), B_{T}(0)\right)
$$

for all $0<S<T \leq T_{0}$, where

$$
\operatorname{Flux}\left(u, M_{S}^{T}\right):=\frac{1}{2} \int_{B_{T}(0) \backslash B_{S}(0)}\left(|\nabla v|^{2}+e^{v^{2}}\right) d y
$$

is the energy flux through $M_{S}^{T}$. Similar identities hold on any region with space-like or null boundary, for instance, in the intersection of a truncated forward light cone with a backward light cone, or with the complement of a backward light cone. 
By (9), in particular, $\lim _{T \downarrow 0} E\left(u(T), B_{T}(0)\right)$ exists and we deduce decay of the flux

$$
\operatorname{Flux}\left(u, M_{0}^{T}\right):=\sup _{0<S<T} \operatorname{Flux}\left(u, M_{S}^{T}\right) \rightarrow 0 \quad \text { as } T \downarrow 0 .
$$

Finally, we also have

$$
E\left(u(T), B_{T}(0)\right) \leq E\left(u\left(T_{0}\right), B_{T_{0}}(0)\right)=: E_{0}
$$

for $0<T<T_{0}$. Set $M^{T}=M_{0}^{T}, K^{T}=K_{0}^{T}$ for brevity.

\subsection{Blow-up criterion}

The work of Ibrahim, Majdoub, and Masmoudi [2] gives rise to the following characterization of blow-up through concentration of energy.

Lemma 2.1. There exists $\varepsilon_{0}>0$ such that

$$
E\left(u(T), B_{T}(0)\right) \geq \varepsilon_{0} \quad \text { for all } 0<T \leq T_{0} .
$$

The short proof of Lemma 2.1 given in [9] also works in the nonsymmetric case.

\subsection{Pointwise estimates}

Without any symmetry assumption we clearly cannot expect to obtain the same pointwise estimates away from $x=0$ that we were able to employ in [9]. However, we can still obtain bounds for the spherical averages

$$
\bar{v}=\bar{v}(t)=\frac{1}{2 \pi} \int_{0}^{2 \pi} v\left(t e^{i \phi}\right) d \phi
$$

of $v$, the trace of $u$ on $M^{T_{0}}$. Indeed, for $0<t<T_{1} \leq T_{0}$ by Hölder's inequality we can bound

$$
\begin{aligned}
|\bar{v}(t)| & \leq\left|\bar{v}\left(T_{1}\right)\right|+\int_{t}^{T_{1}}\left|\bar{v}^{\prime}(s)\right| d s \leq\left|\bar{v}\left(T_{1}\right)\right|+\left(\int_{t}^{T_{1}}|\nabla \bar{v}|^{2} s d s \cdot \int_{t}^{T_{1}} \frac{d s}{s}\right)^{1 / 2} \\
& \leq\left|\bar{v}\left(T_{1}\right)\right|+\pi^{-1 / 2} \operatorname{Flux}^{1 / 2}\left(u, M_{t}^{T_{1}}\right) \log ^{1 / 2}\left(T_{1} / t\right) .
\end{aligned}
$$

In view of (10) we may choose $0<T_{1} \leq \min \left\{1, T_{0}\right\}$ to ensure that for all $0<t \leq T_{1}$,

$$
\operatorname{Flux}^{1 / 2}\left(u, M_{t}^{T_{1}}\right) \leq \operatorname{Flux}^{1 / 2}\left(u, M^{T_{1}}\right) \leq 1 / 8 .
$$

We then fix $0<T_{2} \leq T_{1}$ so that $8\left|\bar{v}\left(T_{1}\right)\right| \leq \log ^{1 / 2}(1 / t)$ for $0<t \leq T_{2}$. Also observing that $\log \left(T_{1} / t\right) \leq \log (1 / t)$ for our choice of $T_{1}$, we thus obtain the bound

$$
4|\bar{v}(t)| \leq \log ^{1 / 2}(1 / t) \quad \text { for all } 0<t \leq T_{2} .
$$




\section{Partial energy decay}

Introduce polar coordinates $(r, \phi)$. The conservation law (7) may then be written in the form

$$
\partial_{t}(r e)-\partial_{r}(r m)=r^{-1} \partial_{\phi}\left(u_{t} u_{\phi}\right)
$$

where now

$$
e=e(u)=\frac{1}{2}\left(u_{t}^{2}+u_{r}^{2}+r^{-2} u_{\phi}^{2}+e^{u^{2}}\right), \quad m=m(u)=u_{t} u_{r} .
$$

Multiplying (1) by $x \cdot \nabla u$ we also obtain the identity

$$
0=\frac{d}{d t}\left(u_{t} x \cdot \nabla u\right)-\operatorname{div}\left(\nabla u x \cdot \nabla u-\frac{x}{2}\left(|\nabla u|^{2}-\left|u_{t}\right|^{2}+e^{u^{2}}\right)\right)+\left|u_{t}\right|^{2}-e^{u^{2}} .
$$

In polar coordinates this reads

$$
\partial_{t}\left(r^{2} u_{t} u_{r}\right)-\frac{1}{2} \partial_{r}\left(r^{2}\left(u_{t}^{2}+u_{r}^{2}-e^{u^{2}}-r^{-2} u_{\phi}^{2}\right)\right)+r\left(u_{t}^{2}-e^{u^{2}}\right)=\partial_{\phi}\left(u_{r} u_{\phi}\right),
$$

that is, we have

$$
\partial_{t}\left(r^{2} m\right)-\partial_{r}\left(r^{2}(e-q)\right)+r\left(u_{t}^{2}-e^{u^{2}}\right)=\partial_{\phi}\left(u_{r} u_{\phi}\right),
$$

where

$$
q=q(u)=r^{-2} u_{\phi}^{2}+e^{u^{2}} .
$$

Finally, we multiply (1) by $u-\bar{v}$ to obtain the equation

$$
0=\frac{d}{d t}\left(u_{t}(u-\bar{v})\right)-\operatorname{div}(\nabla u(u-\bar{v}))+|\nabla u|^{2}-\left|u_{t}\right|^{2}+u_{t} \bar{v}_{t}+u(u-\bar{v}) e^{u^{2}},
$$

that is,

$$
\begin{aligned}
\partial_{t}\left(r u_{t}(u-\bar{v})\right) & -\partial_{r}\left(r u_{r}(u-\bar{v})\right)+r\left(|\nabla u|^{2}-\left|u_{t}\right|^{2}+u_{t} \bar{v}_{t}+u(u-\bar{v}) e^{u^{2}}\right) \\
& =r^{-1} \partial_{\phi}\left((u-\bar{v}) u_{\phi}\right)
\end{aligned}
$$

Multiplying equation (14) by $r / t$, we obtain

$$
\partial_{t}\left(\frac{r^{2}}{t} e\right)-\partial_{r}\left(\frac{r^{2}}{t} m\right)+\frac{r^{2}}{t^{2}} e+\frac{r}{t} m=t^{-1} \partial_{\phi}\left(u_{t} u_{\phi}\right) .
$$

Likewise, upon dividing (15) and (16) by $t$ we find the expressions

$$
\partial_{t}\left(\frac{r^{2}}{t} m\right)-\partial_{r}\left(\frac{r^{2}}{t}(e-q)\right)+\frac{r}{t}\left(u_{t}^{2}-e^{u^{2}}\right)+\frac{r^{2}}{t^{2}} m=t^{-1} \partial_{\phi}\left(u_{r} u_{\phi}\right)
$$


and

$$
\begin{aligned}
\partial_{t}\left(\frac{r}{t} u_{t}(u-\bar{v})\right)- & \partial_{r}\left(\frac{r}{t} u_{r}(u-\bar{v})\right)+\frac{r}{t}\left(|\nabla u|^{2}-\left|u_{t}\right|^{2}+u_{t} \bar{v}_{t}+u_{t} \frac{u-\bar{v}}{t}+u(u-\bar{v}) e^{u^{2}}\right) \\
= & \partial_{t}\left(\frac{r}{t}\left(u_{t}(u-\bar{v})+\frac{|u-\bar{v}|^{2}}{2 t}\right)\right)-\partial_{r}\left(\frac{r}{t} u_{r}(u-\bar{v})\right) \\
& +\frac{r}{t}\left(|\nabla u|^{2}-\left|u_{t}\right|^{2}+u_{t} \bar{v}_{t}+\bar{v}_{t} \frac{u-\bar{v}}{t}+\frac{|u-\bar{v}|^{2}}{t^{2}}+u(u-\bar{v}) e^{u^{2}}\right) \\
= & \frac{1}{r t} \partial_{\phi}\left((u-\bar{v}) u_{\phi}\right),
\end{aligned}
$$

respectively. Dividing both sides of (19) by 2, adding (17), and also adding (18), we then arrive at the equation

$$
\begin{aligned}
& \partial_{t}\left(\frac{r^{2}}{t}(e+\right.\left.\left.m+u_{t} \frac{u-\bar{v}}{2 r}+\frac{|u-\bar{v}|^{2}}{4 r t}\right)\right)-\partial_{r}\left(\frac{r^{2}}{t}\left(e-q+m+u_{r} \frac{u-\bar{v}}{2 r}\right)\right) \\
&+\frac{r}{t}\left(\left(1+\frac{r}{t}\right)(e+m)+\frac{1}{2} u_{t} \bar{v}_{t}+\bar{v}_{t} \frac{u-\bar{v}}{2 t}+\frac{|u-\bar{v}|^{2}}{2 t^{2}}+\frac{u(u-\bar{v})-3}{2} e^{u^{2}}\right) \\
&=t^{-1} \partial_{\phi}\left(\left(u_{r}+u_{t}+\frac{u-\bar{v}}{2 r}\right) u_{\phi}\right)
\end{aligned}
$$

Similarly, subtracting (17) from the sum of (18) and 1/2 times (19), we obtain

$$
\begin{aligned}
& \partial_{t}\left(\frac{r^{2}}{t}\left(m-e+u_{t} \frac{u-\bar{v}}{2 r}+\frac{|u-\bar{v}|^{2}}{4 r t}\right)\right)- \partial_{r}\left(\frac{r^{2}}{t}\left(e-q-m+u_{r} \frac{u-\bar{v}}{2 r}\right)\right) \\
&+\frac{r}{t}\left(\left(1-\frac{r}{t}\right)(e-m)+\frac{1}{2} u_{t} \bar{v}_{t}+\bar{v}_{t} \frac{u-\bar{v}}{2 t}+\frac{|u-\bar{v}|^{2}}{2 t^{2}}+\frac{u(u-\bar{v})-3}{2} e^{u^{2}}\right) \\
&=t^{-1} \partial_{\phi}\left(\left(u_{r}-u_{t}+\frac{u-\bar{v}}{2 r}\right) u_{\phi}\right)
\end{aligned}
$$

In the following we repeatedly make use of Young's inequality $2 a b \leq \delta a^{2}+\delta^{-1} b^{2}$ for any $a, b, \delta>0$. The letter $C$ will denote a generic constant independent of $u, T$, etc., unless otherwise stated. Its value may change from line to line and even within the same line.

Lemma 3.1. For $0<T<\min \left\{T_{2}, e^{-1}\right\}$ we have

$$
\int_{K^{T}}\left(\left(1 \pm \frac{r}{t}\right)(e \pm m)+\frac{|u-\bar{v}|^{2}}{2 t^{2}}+\frac{1}{4}|u-\bar{v}|^{2} e^{u^{2}}\right) \frac{d x d t}{t} \leq C\left(1+E_{0}\right)
$$


Proof. For fixed $0<T<\min \left\{T_{2}, e^{-1}\right\}$ and $0<S<T$ we integrate (20) over the region where $0<S<t<T, 0<r<t, 0 \leq \phi<2 \pi$ corresponding to the truncated cone $K_{S}^{T}$ to obtain

$$
\begin{aligned}
I_{+} & :=\int_{K_{S}^{T}}\left(\left(1+\frac{r}{t}\right)(e+m)+\frac{|u-\bar{v}|^{2}}{2 t^{2}}+\frac{1}{4}|u-\bar{v}|^{2} e^{u^{2}}\right) \frac{d x d t}{t} \\
& =\int_{S}^{T} \int_{0}^{t} \int_{0}^{2 \pi} \frac{r}{t}\left(\left(1+\frac{r}{t}\right)(e+m)+\frac{|u-\bar{v}|^{2}}{2 t^{2}}+\frac{1}{4}|u-\bar{v}|^{2} e^{u^{2}}\right) d \phi d r d t \\
& \leq I I+I I I+I V+V,
\end{aligned}
$$

with $I I, I I I$, and $I V$ corresponding to the boundary terms and with 'error' term

$$
V=-\int_{K_{S}^{T}}\left(\frac{1}{2} u_{t} \bar{v}_{t}+\bar{v}_{t} \frac{u-\bar{v}}{2 t}+\frac{u^{2}-\bar{v}^{2}-6}{4} e^{u^{2}}\right) \frac{d x d t}{t} .
$$

Recalling that $e+m \geq 0$ and using Young's inequality to estimate

$$
\left|u_{t} \frac{u-\bar{v}}{2 t}\right| \leq \frac{1}{2}\left|u_{t}\right|^{2}+\frac{|u-\bar{v}|^{2}}{4 t^{2}} \leq e+\frac{|u-\bar{v}|^{2}}{4 t^{2}}
$$

for any $t$, we can bound the top boundary term:

$$
\begin{aligned}
I I & =-\int_{\{T\} \times B_{T}(0)} \frac{r}{t}\left(e+m+u_{t} \frac{u-\bar{v}}{2 r}+\frac{|u-\bar{v}|^{2}}{4 r t}\right) d x \\
& =-\int_{\{T\} \times B_{T}(0)}\left(\frac{r}{T}(e+m)+u_{t} \frac{u-\bar{v}}{2 T}+\frac{|u-\bar{v}|^{2}}{4 T^{2}}\right) d x \leq \int_{\{T\} \times B_{T}(0)} e d x \leq E_{0} .
\end{aligned}
$$

Also using Poincaré's inequality

$$
\int_{\{t\} \times B_{t}(0)} \frac{|u-\bar{v}|^{2}}{t^{2}} d x \leq C \int_{\{t\} \times B_{t}(0)}|\nabla u|^{2} d x
$$

for any $0<t<T$, in similar fashion we can bound the term corresponding to the lower boundary:

$$
\begin{aligned}
I I I & =\int_{\{S\} \times B_{S}(0)} \frac{r}{t}\left(e+m+u_{t} \frac{u-\bar{v}}{2 r}+\frac{|u-\bar{v}|^{2}}{4 r t}\right) d x \\
& \leq \int_{\{S\} \times B_{S}(0)}\left(\frac{r}{S}(e+m)+e+\frac{|u-\bar{v}|^{2}}{2 S^{2}}\right) d x \leq C \int_{\{S\} \times B_{S}(0)} e d x \leq C E_{0} .
\end{aligned}
$$

Moreover, for the lateral boundary component we have

$$
\begin{aligned}
I V & =\left.\int_{S}^{T} \int_{0}^{2 \pi} \frac{r^{2}}{t}\left(2(e+m)-q+\left(u_{r}+u_{t}\right) \frac{u-\bar{v}}{2 r}+\frac{|u-\bar{v}|^{2}}{4 r t}\right) d \phi d t\right|_{r=t} \\
& =\frac{1}{\sqrt{2}} \int_{M_{S}^{T}}\left(\left(u_{r}+u_{t}\right)^{2}+\left(u_{r}+u_{t}\right) \frac{v-\bar{v}}{2 t}+\frac{|v-\bar{v}|^{2}}{4 t^{2}}\right) d o .
\end{aligned}
$$


1812

Michael Struwe

But again by Poincaré's inequality, for any $0<t<T$ we can estimate

$$
\int_{0}^{2 \pi} \frac{|v-\bar{v}|^{2}}{4 t^{2}} d \phi \leq C \int_{0}^{2 \pi} t^{-2}\left|v_{\phi}\right|^{2} d \phi \leq C \int_{0}^{2 \pi}|\nabla v|^{2} d \phi,
$$

and we conclude that

$$
I V \leq C \operatorname{Flux}\left(u, M^{T}\right) \leq C E_{0} .
$$

Finally, in order to bound $V$, for each $t$ we write

$$
\int_{B_{t}(0)} u_{t} \bar{v}_{t} d x=\int_{B_{t}(0)}\left(\left(u_{t}+u_{r}\right) \bar{v}_{t}-u_{r} \bar{v}_{t}\right) d x
$$

and note that for any $0<\delta<1$ we can bound

$$
\int_{B_{t}(0)}\left|\left(u_{t}+u_{r}\right) \bar{v}_{t}\right| d x \leq \delta \int_{B_{t}(0)}(e+m) d x+\frac{1}{2 \delta} \int_{B_{t}(0)}\left|\bar{v}_{t}\right|^{2} d x .
$$

Next observe that

$$
\begin{aligned}
\int_{B_{t}(0)} u_{r} \bar{v}_{t} d x & =\int_{0}^{2 \pi} \int_{0}^{t}\left(r(u-\bar{v}) \bar{v}_{t}\right)_{r} d r d \phi-\int_{B_{t}(0)} \frac{u-\bar{v}}{r} \bar{v}_{t} d x \\
& =\int_{\partial B_{t}(0)}(v-\bar{v}) \bar{v}_{t} d o-\int_{B_{t}(0)} \frac{u-\bar{v}}{r} \bar{v}_{t} d x,
\end{aligned}
$$

where (23) allows us to bound

$$
\begin{aligned}
\int_{\partial B_{t}(0)}\left|(v-\bar{v}) \bar{v}_{t}\right| d o & \leq \int_{\partial B_{t}(0)} \frac{|v-\bar{v}|^{2}}{2 t} d o+\frac{1}{2} \int_{\partial B_{t}(0)} t\left|\bar{v}_{t}\right|^{2} d o \\
& \leq C t \int_{\partial B_{t}(0)}|\nabla v|^{2} d o+C t^{2}\left|\bar{v}_{t}\right|^{2} .
\end{aligned}
$$

Moreover, we have

$$
\int_{B_{t}(0)}\left|\frac{u-\bar{v}}{r} \bar{v}_{t}\right| d x \leq \delta \int_{B_{t}(0)} \frac{|u-\bar{v}|^{2}}{2 r t} d x+\frac{1}{2 \delta} \int_{B_{t}(0)} \frac{t}{r}\left|\bar{v}_{t}\right|^{2} d x,
$$

with

$$
\frac{1}{2 \delta} \int_{B_{t}(0)} \frac{t}{r}\left|\bar{v}_{t}\right|^{2} d x \leq C \delta^{-1} t^{2}\left|\bar{v}_{t}\right|^{2} .
$$

We split the remaining term

$$
\begin{aligned}
\int_{B_{t}(0)} \frac{|u-\bar{v}|^{2}}{2 r t} d x & \leq \int_{B_{t / 2}(0)} \frac{|u-\bar{v}|^{2}}{2 r t} d x+\int_{B_{t}(0)} \frac{|u-\bar{v}|^{2}}{t^{2}} d x \\
& \leq \int_{B_{t / 2}(0)} \frac{|u-\tilde{u}|^{2}}{r t} d x+C|\tilde{u}-\bar{v}|^{2}+\int_{B_{t}(0)} \frac{|u-\bar{v}|^{2}}{t^{2}} d x
\end{aligned}
$$


where $\tilde{u}=\tilde{u}(t)$ is the average of $u(t)$ on $B_{t / 2}(0)$. Note that we can bound

$$
|\tilde{u}-\bar{v}|^{2} \leq C \int_{B_{t}(0)} \frac{|u-\bar{v}|^{2}}{t^{2}} d x
$$

while by Hölder's inequality and a variant of the Poincaré inequality we have

$$
\int_{B_{t / 2}(0)} \frac{|u-\tilde{u}|^{2}}{2 r t} d x \leq C\left(t^{-2} \int_{B_{t / 2}(0)}|u-\tilde{u}|^{6} d x\right)^{1 / 3} \leq C \int_{B_{t / 2}(0)}|\nabla u|^{2} d x .
$$

Summarizing, we find

$$
\begin{aligned}
\left|\int_{B_{t}(0)} u_{t} \bar{v}_{t} d x\right| \leq & C \delta \int_{B_{t / 2}(0)}|\nabla u|^{2} d x+C \delta \int_{B_{t}(0)}\left(e+m+\frac{|u-\bar{v}|^{2}}{t^{2}}\right) d x \\
& +C t \int_{\partial B_{t}(0)}|\nabla v|^{2} d o+C \delta^{-1} t^{2}\left|\bar{v}_{t}\right|^{2} .
\end{aligned}
$$

Similarly, with the help of Young's inequality we can bound

$$
\int_{B_{t}(0)}\left|\bar{v}_{t} \frac{u-\bar{v}}{t}\right| d x \leq \delta \int_{B_{t}(0)} \frac{|u-\bar{v}|^{2}}{t^{2}} d x+C \frac{1}{2 \delta} \int_{B_{t}(0)}\left|\bar{v}_{t}\right|^{2} d x .
$$

Thus we conclude that

$$
\begin{aligned}
& \left|\int_{K_{S}^{T}}\left(u_{t} \bar{v}_{t}+\bar{v}_{t} \frac{u-\bar{v}}{t}\right) \frac{d x d t}{t}\right| \\
& \leq C \delta \int_{S}^{T} \int_{B_{t / 2}(0)}|\nabla u|^{2} \frac{d x d t}{t}+C \delta I_{+}+C \delta^{-1} \operatorname{Flux}\left(u, M^{T}\right)
\end{aligned}
$$

Finally, we observe that by (13),

$$
\left(6+\bar{v}^{2}-u^{2}\right) e^{u^{2}} \leq\left(6+\bar{v}^{2}\right) e^{6+\bar{v}^{2}} \leq C(6+\log (1 / t)) t^{-1}
$$

for all $0<t<T_{2}$. Thus for $0<T<\min \left\{T_{2}, e^{-1}\right\}$ we have

$$
\int_{K_{S}^{T}}\left(6+\bar{v}^{2}-u^{2}\right) e^{u^{2}} \frac{d x d t}{t} \leq C \int_{K^{T}}(6+\log (1 / t)) \frac{d x d t}{t^{2}} \leq C,
$$

and we conclude that

$$
V \leq C\left(1+\delta I_{+}\right)+C \delta \int_{S}^{T} \int_{B_{t / 2}(0)}|\nabla u|^{2} \frac{d x d t}{t}+C \delta^{-1} \operatorname{Flux}\left(u, M^{T}\right) .
$$

Recalling that Flux $\left(u, M^{T}\right) \leq E_{0}$, together with our estimates for the boundary terms we find

$$
I_{+} \leq C\left(1+\delta I_{+}+\delta^{-1} E_{0}\right)+C \delta \int_{S}^{T} \int_{B_{t / 2}(0)}|\nabla u|^{2} \frac{d x d t}{t} .
$$


The analogous estimate

$$
\begin{aligned}
I_{-} & :=\int_{K_{S}^{T}}\left(\left(1-\frac{r}{t}\right)(e-m)+\frac{|u-\bar{v}|^{2}}{2 t^{2}}+\frac{1}{4}|u-\bar{v}|^{2} e^{u^{2}}\right) \frac{d x d t}{t} \\
& \leq C\left(1+\delta I_{+}+\delta^{-1} E_{0}\right)+C \delta \int_{S}^{T} \int_{B_{t / 2}(0)}|\nabla u|^{2} \frac{d x d t}{t}
\end{aligned}
$$

follows in the same fashion upon integrating (21) over $K_{S}^{T}$.

Finally, we note that we can bound $|\nabla u|^{2} \leq 2 e=(e+m)+(e-m)$ and hence

$$
\int_{S}^{T} \int_{B_{t / 2}(0)}|\nabla u|^{2} \frac{d x d t}{t} \leq I_{+}+2 I_{-} .
$$

Thus for sufficiently small $\delta>0$ with a constant $C$ independent of $S>0$ we have

$$
I_{+}+I_{-} \leq C\left(1+E_{0}\right) \text {. }
$$

Letting $S \downarrow 0$, we obtain the claim.

\section{Proof of Theorem 1.1}

For given $0<\varepsilon<1$ in view of (10) and Lemma 3.1 we may fix $0<T_{\varepsilon}<\min \left\{T_{2}, e^{-1}, \varepsilon^{2}\right\}$ so that

$$
\operatorname{Flux}\left(u, M^{T_{\varepsilon}}\right)+\int_{K^{T_{\varepsilon}}}\left(\left(1 \pm \frac{r}{t}\right)(e \pm m)+\frac{|u-\bar{v}|^{2}}{t^{2}}+|u-\bar{v}|^{2} e^{u^{2}}\right) \frac{d x d t}{t}<\varepsilon .
$$

Introduce the characteristic coordinates

$$
\xi=t+r, \quad \eta=t-r
$$

Then we have

$$
t=\frac{\xi+\eta}{2}, \quad r=\frac{\xi-\eta}{2}
$$

and

$$
\partial_{\xi}=\frac{1}{2}\left(\partial_{t}+\partial_{r}\right), \quad \partial_{\eta}=\frac{1}{2}\left(\partial_{t}-\partial_{r}\right), \quad \partial_{t}=\partial_{\xi}+\partial_{\eta}, \quad \partial_{r}=\partial_{\xi}-\partial_{\eta} .
$$

For any $0<\xi_{1}<T_{\varepsilon}$ let

$$
\Gamma\left(\xi_{1}\right)=\left\{(t, x) \in K^{T_{\varepsilon}} ; \xi=t+|x|=\xi_{1}\right\} .
$$

Integrating (7) over the region

$$
\left\{(t, x) \in K^{T_{\varepsilon}} ; \xi=t+|x| \geq \xi_{1}\right\},
$$


for any such $\xi_{1}$ we obtain

$$
2 \int_{\Gamma\left(\xi_{1}\right)} u_{\eta}^{2} d o \leq \int_{\Gamma\left(\xi_{1}\right)}(e-m) d o \leq E\left(u\left(T_{\varepsilon}\right), B_{T_{\varepsilon}}(0)\right) \leq E_{0}
$$

as a useful variant of the energy inequality (9).

In terms of $\xi$ and $\eta$ we can also write the first two terms in equation (20) in the form

$$
\begin{aligned}
\partial_{t}\left(\frac{r^{2}}{t}\left(e+m+u_{t} \frac{u-\bar{v}}{2 r}+\frac{|u-\bar{v}|^{2}}{4 r t}\right)\right)-\partial_{r}\left(\frac{r^{2}}{t}\left(e-q+m+u_{r} \frac{u-\bar{v}}{2 r}\right)\right) \\
=\partial_{\eta}\left(\frac{r^{2}}{t}\left(2(e+m)-q+u_{\xi} \frac{u-\bar{v}}{r}+\frac{|u-\bar{v}|^{2}}{4 r t}\right)\right) \\
+\partial_{\xi}\left(\frac{r^{2}}{t}\left(q+u_{\eta} \frac{u-\bar{v}}{r}+\frac{|u-\bar{v}|^{2}}{4 r t}\right)\right)
\end{aligned}
$$

Observing that

$$
2(e+m)-q=\left|u_{t}+u_{r}\right|^{2}=4 u_{\xi}^{2}
$$

for $r / t \geq 3 / 4$ we have

$$
\begin{aligned}
& \frac{r}{t}\left(2(e+m)-q+u_{\xi} \frac{u-\bar{v}}{r}+\frac{|u-\bar{v}|^{2}}{4 r t}\right) \\
& =\left(4 \frac{r}{t}-2\right) u_{\xi}^{2}+2\left(u_{\xi}+\frac{u-\bar{v}}{4 t}\right)^{2}+\frac{|u-\bar{v}|^{2}}{8 t^{2}} \geq u_{\xi}^{2}+\frac{|u-\bar{v}|^{2}}{8 t^{2}} .
\end{aligned}
$$

Fix $\lambda_{0}=3 / 4$. Given $0<\xi_{0}<8^{-1} T_{\varepsilon}$, we set $\eta_{0}=\frac{1-\lambda_{0}}{1+\lambda_{0}} \xi_{0}=\xi_{0} / 7$. For $\xi_{1} \in\left[\xi_{0}, 8 \xi_{0}\right]$ we then let

$$
\Gamma_{0}\left(\xi_{1}\right)=\left\{(t, x) \in K^{T_{\varepsilon}} ; \xi=t+|x|=\xi_{1}, \eta=t-|x|<\eta_{0}\right\}
$$

and we define

$$
Q\left(\xi_{1}\right):=\int_{\Gamma_{0}\left(\xi_{1}\right)}\left(q+\frac{|u-\bar{v}|^{2}}{t^{2}}\right) d o .
$$

Note that $t<T_{\varepsilon}$ for any $(t, x)$ with $\xi=t+|x| \leq 8 \xi_{0}<T_{\varepsilon}$. Changing variables $(t, x) \mapsto(\xi=t+|x|, x)$, we see that for any $\xi_{1}<T_{\varepsilon} / 2$, with an absolute constant $C$,

$$
\begin{aligned}
\inf _{\xi_{1}<\xi<2 \xi_{1}} Q(\xi) & \leq \xi_{1}^{-1} \int_{\xi_{1}}^{2 \xi_{1}} Q(\xi) d \xi \\
& \leq C \int_{K^{T_{\varepsilon}}}\left(\left(1+\frac{r}{t}\right)(e+m)+\frac{|u-\bar{v}|^{2}}{2 t^{2}}\right) \frac{d x d t}{t}<C \varepsilon .
\end{aligned}
$$

Thus, we can choose numbers $\xi_{1} \in\left[\xi_{0}, 2 \xi_{0}\right]$ and $\xi_{2} \in\left[4 \xi_{0}, 8 \xi_{0}\right]$ such that

$$
Q\left(\xi_{1}\right) \leq 2 \inf _{\xi_{0}<\xi<2 \xi_{0}} Q(\xi)<C \varepsilon, \quad Q\left(\xi_{2}\right) \leq 2 \inf _{4 \xi_{0}<\xi<8 \xi_{0}} Q(\xi)<C \varepsilon .
$$


Lemma 4.1. For any $0<\xi_{0}<8^{-1} T_{\varepsilon}$, and any $\xi_{1} \in\left[\xi_{0}, 2 \xi_{0}\right], \xi_{2} \in\left[4 \xi_{0}, 8 \xi_{0}\right]$ as above,

$$
\sup _{2 \xi_{0}<\xi<4 \xi_{0}} Q(\xi) \leq \sup _{\xi_{1}<\xi<\xi_{2}} Q(\xi)<C \sqrt{\varepsilon} .
$$

Proof. Consider the set

$$
R=R\left(\xi_{1}, \xi_{2}\right)=\left\{(t, x) \in K^{T_{\varepsilon}} ; \xi_{1}<\xi<\xi_{2}, 0<\eta<\eta_{0}\right\}
$$

with boundary $\partial R=\bigcup_{i=1}^{4} \Gamma_{i}$, where

$$
\begin{array}{ll}
\Gamma_{1}=\left\{(t, x) ; \xi_{1}<\xi<\xi_{2}, \eta=0\right\}, & \Gamma_{2}=\Gamma_{0}\left(\xi_{2}\right), \\
\Gamma_{3}=\left\{(t, x) ; \xi_{1}<\xi<\xi_{2}, \eta=\eta_{0}\right\}, & \Gamma_{4}=\Gamma_{0}\left(\xi_{1}\right) .
\end{array}
$$

Integrating (20) over $R$, we find the identity

$$
A_{0}+A_{3}=A_{1}-A_{2}+A_{4}+V
$$

where

$$
A_{0}=\int_{R}\left(\left(1+\frac{r}{t}\right)(e+m)+\frac{|u-\bar{v}|^{2}}{2 t^{2}}+\frac{1}{4}|u-\bar{v}|^{2} e^{u^{2}}\right) \frac{d x d t}{t}
$$

and where the terms $A_{i}, 1 \leq i \leq 4$, correspond to integrals over the boundary components $\Gamma_{i}, 1 \leq i \leq 4$. Finally, $V$ again denotes the 'error' term

$$
V=-\int_{R}\left(\frac{1}{2} u_{t} \bar{v}_{t}+\bar{v}_{t} \frac{u-\bar{v}}{2 t}+\frac{u^{2}-\bar{v}^{2}-6}{4} e^{u^{2}}\right) \frac{d x d t}{t} .
$$

By (26),

$$
0 \leq A_{0} \leq \varepsilon .
$$

Moreover, using (28), (29), and (23) we find

$$
\begin{aligned}
A_{1} & =\int_{\Gamma_{1}}\left(\left(4 \frac{r}{t}-2\right) u_{\xi}^{2}+2\left(u_{\xi}+\frac{u-\bar{v}}{4 t}\right)^{2}+\frac{|u-\bar{v}|^{2}}{8 t^{2}}\right) d o \\
& \leq C \int_{\Gamma_{1}}\left(u_{\xi}^{2}+\frac{|v-\bar{v}|^{2}}{t^{2}}\right) d o \leq C \operatorname{Flux}\left(u, M^{8 \xi_{0}}\right) \leq C \varepsilon .
\end{aligned}
$$

Using Young's inequality to bound

$$
\left|u_{\eta} \frac{u-\bar{v}}{2 t}\right| \leq \frac{\delta}{2} u_{\eta}^{2}+\frac{|u-\bar{v}|^{2}}{8 \delta t^{2}}
$$

and recalling that the energy inequality (27) allows us to bound

$$
2 \int_{\Gamma_{0}(\xi)} u_{\eta}^{2} d o \leq E_{0}
$$


for any $\xi<8 \xi_{0}$, we also find

$$
\left|A_{2}\right|=\left|\int_{\Gamma_{0}\left(\xi_{2}\right)}\left(\frac{r}{t} q+u_{\eta} \frac{u-\bar{v}}{2 t}+\frac{|u-\bar{v}|^{2}}{4 t^{2}}\right) d o\right| \leq\left(1+\delta^{-1}\right) Q\left(\xi_{2}\right)+\delta E_{0},
$$

and similarly for $A_{4}$. Choosing $\delta=\sqrt{\varepsilon}$, by choice of $\xi_{1}$ and $\xi_{2}$ we obtain

$$
\left|A_{2}\right|<C \sqrt{\varepsilon}, \quad\left|A_{4}\right|<C \sqrt{\varepsilon} .
$$

In order to proceed, observe that by (29) we have

$$
A_{3} \geq \int_{\Gamma_{3}}\left(u_{\xi}^{2}+\frac{|u-\bar{v}|^{2}}{8 t^{2}}\right) d o .
$$

The error term $V$ can then be bounded as in the proof of Lemma 3.1, on noting that we can express

$$
\int_{R} u_{r} \bar{v}_{t} \frac{d x d t}{t}=\int_{R}\left(r(u-\bar{v}) \bar{v}_{t}\right)_{r} \frac{d r d \phi d t}{t}-\int_{R} \frac{u-\bar{v}}{r} \bar{v}_{t} \frac{d x d t}{t}
$$

with

$$
\begin{aligned}
\left|\int_{R}\left(r(u-\bar{v}) \bar{v}_{t}\right)_{r} \frac{d r d \phi d t}{t}\right| & \leq \int_{\partial R}\left|(u-\bar{v}) \bar{v}_{t}\right| \frac{d o}{t} \leq \delta \int_{\partial R} \frac{|u-\bar{v}|^{2}}{8 t^{2}} d o+\frac{2}{\delta} \int_{\partial R}\left|\bar{v}_{t}\right|^{2} d o \\
& \leq \delta\left(A_{3}+Q\left(\xi_{1}\right)+Q\left(\xi_{2}\right)\right)+C \delta^{-1} \operatorname{Flux}\left(u, M^{4 \xi_{0}}\right) \\
& \leq \delta A_{3}+C \varepsilon+C \delta^{-1} \varepsilon
\end{aligned}
$$

for any $0<\delta<1$, in view of (23), (26), and our bounds for $Q\left(\xi_{1}\right)$ and $Q\left(\xi_{2}\right)$. Also note that in view of the fact that $r / t \geq \lambda_{0}=3 / 4$ on $R$ we do not need to perform step (24); instead, we can easily estimate

$$
\begin{aligned}
\int_{R} \frac{|u-\bar{v}|}{r}\left|\bar{v}_{t}\right| \frac{d x d t}{t} & \leq 2 \int_{R} \frac{|u-\bar{v}|}{t}\left|\bar{v}_{t}\right| \frac{d x d t}{t} \leq \int_{R} \frac{|u-\bar{v}|^{2}}{2 t^{2}} \frac{d x d t}{t}+2 \int_{R}\left|\bar{v}_{t}\right|^{2} \frac{d x d t}{t} \\
& \leq A_{0}+C \operatorname{Flux}\left(u, M^{4 \xi_{0}}\right) \leq C \varepsilon .
\end{aligned}
$$

Finally, recalling that $T_{\varepsilon}<1$, in view of (13) we can estimate

$$
\left(6+\bar{v}^{2}-u^{2}\right) e^{u^{2}} \leq\left(6+\bar{v}^{2}\right) e^{6+\bar{v}^{2}} \leq C(6+\log (1 / t)) t^{-1 / 2}
$$

for all $0<t<T_{\varepsilon}$. Hence we have

$$
\int_{R}\left(6+\bar{v}^{2}-u^{2}\right) e^{u^{2}} \frac{d x d t}{t} \leq C \int_{K^{8 \xi_{0}}}(6+\log (1 / t)) \frac{d x d t}{t^{3 / 2}} \leq C \sqrt{\xi_{0}} \leq C \varepsilon .
$$

Together with (33), when choosing $\delta=1 / 2$, we thus obtain the bound

$$
V \leq \frac{1}{2} A_{3}+C \varepsilon
$$


From (30) we then conclude that

$$
0 \leq A_{3} \leq C \sqrt{\varepsilon}
$$

Thus for any $\xi_{1}<\xi<\xi_{2}$, when integrating (20) over $R\left(\xi_{1}, \xi\right)$, from the analogue of (30) and choosing a sufficiently small number $\delta>0$ in (33) we now find that

$$
A_{2}(\xi):=\int_{\Gamma_{0}(\xi)}\left(\frac{r}{t} q+u_{\eta} \frac{u-\bar{v}}{t}+\frac{|u-\bar{v}|^{2}}{4 t^{2}}\right) d o \leq C \sqrt{\varepsilon}+\frac{1}{2} Q(\xi) .
$$

This estimate implies the desired bound for $Q(\xi)$ once we control the middle term. But by Hölder's inequality, for any $\xi_{1}<\xi<\xi_{2}$ we have

$$
\begin{aligned}
|(u-\bar{v})(\xi)|^{2} & \leq\left(\left|(u-\bar{v})\left(\xi_{1}\right)\right|+\int_{\xi_{1}}^{\xi_{2}}\left|u_{\xi}-\bar{v}_{\xi}\right| d \xi\right)^{2} \\
& \leq 2\left|(u-\bar{v})\left(\xi_{1}\right)\right|^{2}+2\left(\xi_{2}-\xi_{1}\right) \int_{\xi_{1}}^{\xi_{2}}\left|u_{\xi}-\bar{v}_{\xi}\right|^{2} d \xi .
\end{aligned}
$$

Integrating over $\Gamma_{0}(\xi)$, observing that the surface measure $d o$ may be expressed as $r d \eta d \phi$, where $r=(\xi-\eta) / 2$, and noting that throughout $R$ we have $0 \leq \eta \leq \eta_{0}=\xi_{0} / 7$, $\xi_{0} \leq \xi \leq 2 t \leq 16 \xi_{0}$, we obtain

$$
\begin{aligned}
\int_{\Gamma_{0}(\xi)} \frac{|u-\bar{v}|^{2}}{t^{2}} d o & \leq C \int_{\Gamma_{0}\left(\xi_{1}\right)} \frac{|u-\bar{v}|^{2}}{t^{2}} d o+C \int_{R}\left(|u \xi|^{2}+\left|\bar{v}_{t}\right|^{2}\right) \frac{d x d t}{t} \\
& \leq C \varepsilon+C A_{0}+C \operatorname{Flux}\left(u, M^{8 \xi_{0}}\right) \leq C \varepsilon
\end{aligned}
$$

for any $\xi_{1}<\xi<\xi_{2}$. By (31) and (32), again choosing $\delta=\sqrt{\varepsilon}$, we can estimate

$$
\begin{aligned}
A_{2}(\xi) & =\int_{\Gamma_{0}(\xi)}\left(\frac{r}{t} q+\left(u_{t}-u_{r}\right) \frac{u-\bar{v}}{2 t}+\frac{|u-\bar{v}|^{2}}{4 t^{2}}\right) d o \\
& \geq \lambda_{0} Q(\xi)-\left(\lambda_{0}+\frac{1}{8 \delta}\right) \int_{\Gamma_{0}(\xi)} \frac{|u-\bar{v}|^{2}}{t^{2}} d o-\frac{\delta}{2} \int_{\Gamma_{0}(\xi)} u_{\eta}^{2} d o \geq \frac{3}{4} Q(\xi)-C \sqrt{\varepsilon}
\end{aligned}
$$

Together with (34) it follows that

$$
\sup _{\xi_{1}<\xi<\xi_{2}} Q(\xi)<C \sqrt{\varepsilon}
$$

as claimed.

Combining Lemmas 3.1 and 4.1 we can bound the nonlinear term in equation (1) in any $L^{p}$-norm. A key role is played by the following improvement of the Moser-Trudinger inequality (5). 
Lemma 4.2. For any $E>0$ and $p<\infty$ there exists a number $\varepsilon=4 \pi^{2} /\left(p^{2} E\right)>0$ and a constant $C>0$ such that for any $\xi_{0}>0$ and $v \in H_{0}^{1}\left([0,1]^{2}\right)$ with

$$
\int_{0}^{1} \int_{0}^{1}\left(\xi_{0}\left|v_{y}\right|^{2}+\xi_{0}^{-1}\left|v_{x}\right|^{2}\right) d x d y \leq E, \quad \int_{0}^{1} \int_{0}^{1} \xi_{0}^{-1}\left|v_{x}\right|^{2} d x d y \leq \varepsilon
$$

we have

$$
\int_{0}^{1} \int_{0}^{1} e^{p v^{2}} d x d y \leq C .
$$

Proof. Given $v \in H_{0}^{1}\left([0,1]^{2}\right)$ as above, set $\alpha=\left(\xi_{0}^{2} \varepsilon / E\right)^{1 / 4}>0$ and let

$$
v_{\alpha}(x, y)=v(x / \alpha, \alpha y) \in H_{0}^{1}([0, \alpha] \times[0,1 / \alpha]),
$$

satisfying

$$
\begin{aligned}
\int_{0}^{\alpha} \int_{0}^{1 / \alpha}\left|\nabla v_{\alpha}\right|^{2} d x d y & =\int_{0}^{1} \int_{0}^{1}\left(\alpha^{-2}\left|v_{x}\right|^{2}+\alpha^{2}\left|v_{y}\right|^{2}\right) d x d y \\
& \leq \varepsilon \xi_{0} \alpha^{-2}+\alpha^{2} E / \xi_{0}=2(\varepsilon E)^{1 / 2}=4 \pi / p
\end{aligned}
$$

by our choice of $\varepsilon$. Note that the map $(x, y) \mapsto(x / \alpha, \alpha y)$ is measure-preserving; in particular, for any $s \geq 0$,

$$
\left|\left\{(x, y) ; v_{\alpha}^{2}(x, y) \geq s\right\}\right|=\left|\left\{(x, y) ; v^{2}(x, y) \geq s\right\}\right|,
$$

and

$$
\int_{0}^{1} \int_{0}^{1} e^{p v^{2}} d x d y=\int_{0}^{\alpha} \int_{0}^{1 / \alpha} e^{p v_{\alpha}^{2}} d x d y .
$$

But by (36), with the constant $C(4 \pi)$ in (5), we have

$$
\int_{0}^{\alpha} \int_{0}^{1 / \alpha} e^{p v_{\alpha}^{2}} d x d y \leq C(4 \pi)
$$

and our claim follows.

Lemma 4.3. There exists $\varepsilon>0$ and a constant $C<\infty$ such that for any $0<T<$ $4^{-1} T_{\varepsilon}$,

$$
\int_{K^{T}} e^{4 u^{2}} d x d t \leq C T
$$

Proof. Given $0<\xi_{0}<8^{-1} T_{\varepsilon}$, let $\eta_{0}=\frac{1-\lambda_{0}}{1+\lambda_{0}} \xi_{0}$, where $\lambda_{0}=3 / 4$ as before. For $\xi_{0}<$ $\xi_{4} \leq 8 \xi_{0}$ recall the definitions

$$
\begin{aligned}
\Gamma\left(\xi_{4}\right) & =\left\{(t, x) \in K^{T_{\varepsilon}} ; \xi=t+|x|=\xi_{4}\right\} \\
\Gamma_{0}\left(\xi_{4}\right) & =\left\{(t, x) \in K^{T_{\varepsilon}} ; \xi=t+|x|=\xi_{4}, \eta=t-|x|<\eta_{0}\right\}
\end{aligned}
$$


from the beginning of this section. Also let

$$
\begin{aligned}
& \Gamma_{1}\left(\xi_{4}\right)=\left\{(t, x) \in K^{T_{\varepsilon}} ; \xi=t+|x|=\xi_{4}, \eta=t-|x| \geq 3 \eta_{0} / 4\right\}, \\
& \Gamma_{2}\left(\xi_{4}\right)=\left\{(t, x) \in K^{T_{\varepsilon}} ; \xi=t+|x|=\xi_{4}, \eta=t-|x| \geq \eta_{0} / 2\right\} .
\end{aligned}
$$

With the help of Lemma 4.2 and (38) below, respectively, for any fixed $0<\xi_{0}<8^{-1} T_{\varepsilon}$ as above and any $2 \xi_{0} \leq \xi \leq 4 \xi_{0}$ we now bound the integral of $e^{4 u^{2}}$ over $\Gamma(\xi)$, uniformly in $\xi$. Note that for each such $\xi$ we have $\Gamma(\xi) \subset \Gamma_{0}(\xi) \cup \Gamma_{1}(\xi)$.

First consider $\Gamma_{1}(\xi) \subset \Gamma_{2}(\xi)$. Note that we have $r / t \leq 1-v_{0}<1$ throughout $\Gamma_{2}(\xi)$ for any $\xi_{0} \leq \xi \leq 8 \xi_{0}$, with a uniform constant $\nu_{0}>0$ determined by our choice of $\lambda_{0}$. By Fubini's theorem and in view of (26) for each $0<\xi_{0}<8^{-1} T_{\varepsilon}$ there is $\xi_{4} \in\left[4 \xi_{0}, 8 \xi_{0}\right]$ such that

$$
\begin{aligned}
\int_{\Gamma_{2}\left(\xi_{4}\right)}\left(v_{0}(e-m)+\right. & \left.\frac{|u-\bar{v}|^{2}}{2 t^{2}}\right) d o \\
& \leq \int_{\Gamma_{2}\left(\xi_{4}\right)}\left(\left(1-\frac{r}{t}\right)(e-m)+\frac{|u-\bar{v}|^{2}}{2 t^{2}}\right) d o \\
& \leq 2 \inf _{4 \xi_{0}<\xi<8 \xi_{0}} \int_{\Gamma_{2}(\xi)}\left(\left(1-\frac{r}{t}\right)(e-m)+\frac{|u-\bar{v}|^{2}}{2 t^{2}}\right) d o \\
& \leq C \int_{K^{T_{\varepsilon}}}\left(\left(1-\frac{r}{t}\right)(e-m)+\frac{|u-\bar{v}|^{2}}{2 t^{2}}\right) \frac{d x d t}{t} \leq C \varepsilon
\end{aligned}
$$

In particular, we have

$$
\int_{\Gamma_{2}\left(\xi_{4}\right)}(e-m) d o \leq C \varepsilon .
$$

Upon integrating the conservation law (7) over the region

$$
\left\{(t, x) \in K^{T_{\varepsilon}} ; \xi_{3} \leq \xi=t+|x| \leq \xi_{4}, \eta=t-|x| \geq \eta_{0} / 2\right\}
$$

for any $\xi_{3} \in\left[2 \xi_{0}, 4 \xi_{0}\right]$, we then also obtain

$$
\sup _{2 \xi_{0}<\xi<4 \xi_{0}} \int_{\Gamma_{2}(\xi)}(e-m) d o \leq C \varepsilon .
$$

Estimating as in (35), from (26) and (37) for any $2 \xi_{0}<\xi<4 \xi_{0}$ we likewise find the estimate

$$
\begin{aligned}
\int_{\Gamma_{2}(\xi)} \frac{|u-\bar{v}|^{2}}{t^{2}} d o & \leq C \int_{\Gamma_{2}\left(\xi_{4}\right)} \frac{|u-\bar{v}|^{2}}{t^{2}} d o+C \int_{K^{T_{\varepsilon}}}\left(|u \xi|^{2}+\left|\bar{v}_{t}\right|^{2}\right) \frac{d x d t}{t} \\
& \leq C \varepsilon+C \operatorname{Flux}\left(u, M^{\xi_{0}}\right) \leq C \varepsilon
\end{aligned}
$$

Hence we obtain the uniform bound

$$
\sup _{2 \xi_{0}<\xi<4 \xi_{0}} \int_{\Gamma_{2}(\xi)}\left((e-m)+\frac{|u-\bar{v}|^{2}}{t^{2}}\right) d o \leq C \varepsilon .
$$


Fix a smooth cut-off function $0 \leq \varphi_{1} \leq 1$ on $\mathbb{R}$ such that $\varphi_{1}(\eta)=1$ for $\eta \geq 3 \eta_{0} / 4$ and $\varphi_{1}(\eta)=0$ for $\eta \leq \eta_{0} / 2$, with $\left|\varphi_{1}^{\prime}\right| \leq 8 / \eta_{0} \leq C / \xi_{0}$, and set $u_{1}=\varphi_{1}(\eta)(u-\bar{v})$. For a point $z \in \Gamma(\xi)$ write $z=(t, y)=(\xi-|y|, y)$ with $y=r e^{i \phi} \in B_{\xi / 2}(0)$. Note that $\eta=t-r=\xi-2|y|$. Letting $v_{1}(y)=u_{1}(\xi-|y|, y) \in H_{0}^{1}\left(B_{\xi / 2}(0)\right)$, for sufficiently small $\varepsilon>0$ by (38) we have

$$
\begin{aligned}
\int_{B_{\xi / 2}(0)}\left|\nabla v_{1}\right|^{2} d y & =\int_{\Gamma_{2}(\xi)}\left(4\left|\partial_{\eta} u_{1}\right|^{2}+r^{-2}\left|\partial_{\phi} u_{1}\right|^{2}\right) d o \\
& \leq C \int_{\Gamma_{2}(\xi)}\left((e-m)+\frac{|u-\bar{v}|^{2}}{t^{2}}\right) d o \leq C \varepsilon \leq \pi / 4,
\end{aligned}
$$

uniformly in $2 \xi_{0}<\xi<4 \xi_{0}$. In view of (5) it follows that

$$
\int_{\Gamma_{1}(\xi)} e^{16|u-\bar{v}|^{2}} d x \leq \int_{\Gamma_{2}(\xi)} e^{16 u_{1}^{2}} d o \leq C \int_{B_{\xi / 2}(0)} e^{16 v_{1}^{2}} d y \leq C,
$$

uniformly in $2 \xi_{0}<\xi<4 \xi_{0}$, with absolute constants $C>0$.

Also let $0 \leq \varphi_{2}=\varphi_{2}(\eta) \leq 1$ be a smooth cut-off function such that $\varphi_{2}(\eta)=1$ for $\eta \leq 3 \eta_{0} / 4$ and $\varphi_{2}(\eta)=0$ for $\eta \geq \eta_{0}$, with $\left|\varphi_{2}^{\prime}\right| \leq 8 / \eta_{0} \leq C / \xi_{0}$. Finally, fix a smooth cut-off function $0 \leq \chi=\chi(\phi) \leq 1$ satisfying $\chi(\phi)=1$ for $|\phi| \leq \pi / 8$ and $\chi(\phi)=0$ for $|\phi| \geq \pi / 4$. Set $u_{2}=\varphi_{2}(\eta)(u-\bar{v})$. After extending $u_{2}(\xi, \eta, \phi)=u_{2}(\xi,-\eta, \phi)$ for $\eta<0$ for fixed $\xi$, also let $u_{2 k}=u_{2 k}(\xi, \eta, \phi)=\chi(\phi-k \pi / 4) u_{2}, 1 \leq k \leq 8$. Note that $u_{2 k}(\xi, \cdot, \cdot) \in H_{0}^{1}\left(\left[-\eta_{0}, \eta_{0}\right] \times[(k-1) \pi / 4,(k+1) \pi / 4]\right), 1 \leq k \leq 8$, and we have

$$
\begin{aligned}
& \int_{-\eta_{0}}^{\eta_{0}} \int_{(k-1) \pi / 4}^{(k+1) \pi / 4}\left(\left|\partial_{\eta} u_{2 k}\right|^{2}+r^{-2}\left|\partial_{\phi} u_{2 k}\right|^{2}\right) r d \phi d \eta \\
& \quad \leq C \int_{\Gamma_{0}(\xi)}\left((e-m)+\frac{|u-\bar{v}|^{2}}{t^{2}}\right) d o \leq C E_{0}
\end{aligned}
$$

whereas Lemma 4.1 yields the bound

$$
\begin{array}{rl}
\int_{-\eta_{0}}^{\eta_{0}} \int_{(k-1) \pi / 4}^{(k+1) \pi / 4}\left(r^{-2}\left|\partial_{\phi} u_{2 k}\right|^{2}\right) r & d \phi d \eta \\
\leq C \int_{\Gamma_{0}(\xi)}\left(q+\frac{|u-\bar{v}|^{2}}{t^{2}}\right) d o=C Q(\xi) \leq C \sqrt{\varepsilon}
\end{array}
$$

uniformly in $1 \leq k \leq 8$ and $2 \xi_{0}<\xi<4 \xi_{0}$. Also observe that

$$
\frac{\xi_{0} \lambda_{0}}{1+\lambda_{0}}=\frac{\xi_{0}-\eta_{0}}{2} \leq r=\frac{\xi-\eta}{2} \leq 2 \xi_{0}+\eta_{0} / 2 \leq 3 \xi_{0}
$$

for $2 \xi_{0}<\xi<4 \xi_{0}$ and $|\eta| \leq \eta_{0}$. Thus by Lemma 4.2 for sufficiently small $\varepsilon>0$ with an absolute constant $C>0$ we find that

$$
\sup _{2 \xi_{0}<\xi<4 \xi_{0}} \int_{\Gamma_{0}(\xi)} e^{16 u_{2 k}^{2}} d x \leq C
$$

uniformly in $1 \leq k \leq 8$. 
Now observe that $|u| \leq|u-\bar{v}|+|\bar{v}| \leq 2 \max \{|u-\bar{v}|,|\bar{v}|\}$. Thus, by choice of $\varphi_{1}$, $\varphi_{2}$, and $\chi$, and in view of (11), we can bound

$$
\begin{aligned}
\int_{\Gamma(\xi)} e^{4 u^{2}} d o & \leq \int_{\Gamma(\xi)} e^{16|u-\bar{v}|^{2}} d o+\int_{\Gamma(\xi)} e^{16|\bar{v}|^{2}} d o \\
& \leq \int_{\Gamma_{1}(\xi)} e^{16 u_{1}^{2}} d o+\sum_{1 \leq k \leq 8} \int_{\Gamma_{0}(\xi)} e^{16 u_{2 k}^{2}} d o+\int_{\Gamma(\xi)} t^{-1} d o \leq C,
\end{aligned}
$$

uniformly in $2 \xi_{0}<\xi<4 \xi_{0}$. Hence for any $0<\xi_{0}<8^{-1} T_{\varepsilon}$ with a constant $C$ independent of $\xi_{0}$ we find

$$
\int_{2 \xi_{0}}^{4 \xi_{0}} \int_{\Gamma(\xi)} e^{4 u^{2}} d o d \xi \leq C \xi_{0}
$$

Note that the collection $(\Gamma(\xi))_{0<\xi<4 \xi_{0}}$ covers the cone $K^{2 \xi_{0}}$. Replacing $\xi_{0}$ by $2^{-k} \xi_{0}$ and adding the resulting estimates, after the change of variables $(t, x) \mapsto(\xi=t+|x|, x)$ we then obtain

$$
\int_{K^{2 \xi_{0}}} e^{4 u^{2}} d x d t \leq \sum_{k \in \mathbb{N}_{0}} \int_{2^{1-k} \xi_{0}}^{2^{2-k} \xi_{0}} \int_{\Gamma(\xi)} e^{4 u^{2}} d o d \xi \leq C \sum_{k \in \mathbb{N}_{0}} 2^{-k} \xi_{0} \leq C \xi_{0},
$$

as desired.

Proof of Theorem 1.1. Fix $\varepsilon>0,0<T \leq 4^{-1} T_{\varepsilon}$ as in Lemma 4.3 and let $u^{(0)}$ be the solution to the homogeneous wave equation $u_{t t}^{(0)}-\Delta u^{(0)}=0$ in $K^{T}$ with initial data $u^{(0)}(T)=u(T), u_{t}^{(0)}(T)=u_{t}(T)$. Multiplying the equation

$$
\left(u-u^{(0)}\right)_{t t}-\Delta\left(u-u^{(0)}\right)+u e^{u^{2}}=0
$$

with $\left(u-u^{(0)}\right)_{t}$ and integrating over $K_{S}^{T}$, we obtain the estimate

$$
\begin{aligned}
\frac{1}{2} \int_{B_{S}(0)} & \left|D\left(u-u^{(0)}\right)(S)\right|^{2} d x \leq \int_{K_{S}^{T}(0)}\left|\left(u-u^{(0)}\right)_{t}\right||u| e^{u^{2}} d x d t \\
& \leq\left(\sup _{S \leq t \leq T} \int_{B_{t}(0)}\left|D\left(u-u^{(0)}\right)(t)\right|^{2} d x\right)^{1 / 2}\left(T \int_{K_{S}^{T}(0)} u^{2} e^{2 u^{2}} d x d t\right)^{1 / 2}
\end{aligned}
$$

where $D=\left(\partial_{t}, \nabla\right)$. Replacing $S$ by a suitable $t \in[S, T]$, we arrive at

$$
\sup _{S \leq t \leq T} \int_{B_{t}(0)}\left|D\left(u-u^{(0)}\right)(t)\right|^{2} d x \leq 4 T \int_{K_{S}^{T}(0)} u^{2} e^{2 u^{2}} d x d t \leq 4 T \int_{K^{T}(0)} e^{4 u^{2}} d x d t .
$$

But choosing $T>0$ sufficiently small, in view of Lemma 4.3 we can achieve that

$$
4 T \int_{K^{T}\left(z_{0}\right)} e^{4 u^{2}} d x d t<\varepsilon_{0},
$$


where $\varepsilon_{0}>0$ is the constant defined in Lemma 2.1. Since

$$
\lim _{t \downarrow 0} \int_{B_{t}(0)}\left|D u^{(0)}(t)\right|^{2} d x=0
$$

and since by Lemma 3.1 we also have

$$
\liminf _{t \downarrow 0} \int_{B_{t}(0)} e^{u(t)^{2}} d x=0,
$$

we then find that

$$
\liminf _{t \downarrow 0} E\left(u(t), B_{t}(0)\right)<\varepsilon_{0},
$$

contradicting Lemma 2.1. The proof is complete.

\section{References}

[1] Ibrahim, S., Jrad, R.: Strichartz type estimates and the wellposedness of an energy critical 2d wave equation in a bounded domain. J. Differential Equations 250, 3740-3771 (2011) Zbl 1218.35146 MR 2773184

[2] Ibrahim, S., Majdoub, M., Masmoudi, N.: Global solutions for a semilinear, two-dimensional Klein-Gordon equation with exponential-type nonlinearity. Comm. Pure Appl. Math. 59, 1639-1658 (2006) Zbl 1117.35049 MR 2254447

[3] Ibrahim, S., Majdoub, M., Masmoudi, N.: Well- and ill-posedness issues for energy supercritical waves. Anal. PDE 4, 341-367 (2011) Zbl pre06165651 MR 2859857

[4] Ibrahim, S., Majdoub, M., Masmoudi, N., Nakanishi, K.: Scattering for the two-dimensional energy-critical wave equation. Duke Math. J. 150, 287-329 (2009) Zbl 1206.35175 MR 2569615

[5] Lebeau, G.: Non linear optic and supercritical wave equation. Bull. Soc. Roy. Sci. Liège 70, 267-306 (2001) Zbl 1034.35137 MR 1904059

[6] Moser, J.: A sharp form of an inequality by N. Trudinger. Indiana Univ. Math. J. 20, 10771092 (1971) Zbl 0203.43701 MR 0301504

[7] Shatah, J., Struwe, M.: Geometric Wave Equations. Courant Lecture Notes 2, New York Univ. (1998), 2nd ed., Amer. Math. Soc. (2000) Zbl 0993.35001 MR 1674843

[8] Struwe, M.: On uniqueness and stability for supercritical nonlinear wave and Schrdinger equations. Int. Math. Res. Notices 2006, art. ID 76737, 14 pp. Zbl 1115.35088 MR 2211155

[9] Struwe, M.: Global well-posedness of the Cauchy problem for a super-critical nonlinear wave equation in 2 space dimensions. Math. Ann. 350, 707-719 (2011) Zbl 1227.35216 MR 2805642

[10] Tao, T.: Global regularity for a logarithmically supercritical defocusing nonlinear wave equation for spherically symmetric data. J. Hyperbolic Differential Equations 4, 259-265 (2007) Zbl 1124.35043 MR 2329385

[11] Trudinger, N. S.: On embeddings into Orlicz spaces and some applications. J. Math. Mech. 17, 473-484 (1967) Zbl 0163.36402 MR 0216286 